\title{
THE AGRICULTURAL SURVEY
}

\author{
OF
}

AFGHANISTAN

$+$ 


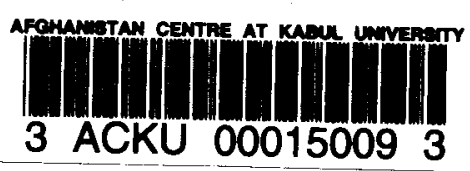

KUNAR

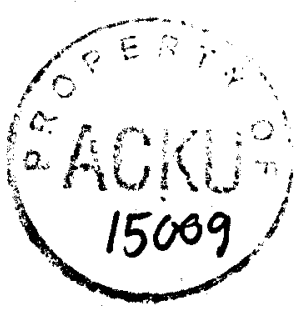




\section{AGRICULTURAL SURVEY OF AFGHANISTAN}

\section{GENERAL AGRICULTURAL INFORMATION ON KUNAR}

Prepared for MIke McGovern

Date: July 9,1989

Information in section one and two was collected in Summer 1987.

No. of Farming households. surveyed: 549

Surveyed in Pakistan:

238

Surveyed in Afghanistan:

218

The numbers of farmers questionned differ from table to table as some farmers did not answer all the questions.

Topics covered:

\section{SECTION ONE \\ DISTRICT DATA SPLIT BETWEEN FARMERS IN CAMPS AND AFGHANISTAN}

1. Farm size distribution table

2. Farm size

2c Farm size, further detail

$3 a$ Areas and yields of principle crops

3b Numbers of farmers growing principle crops

4. Methods of land preparation (Farm power)

4E. Fertiliser

5. Seed

7A. Average number of trained oxen

7B. Average number of farmers with other animals

7C. Average size of cattle herd for farmers

9. Direct effects of war

10. Greatest farming problems 
1978 Farm Size Distribution

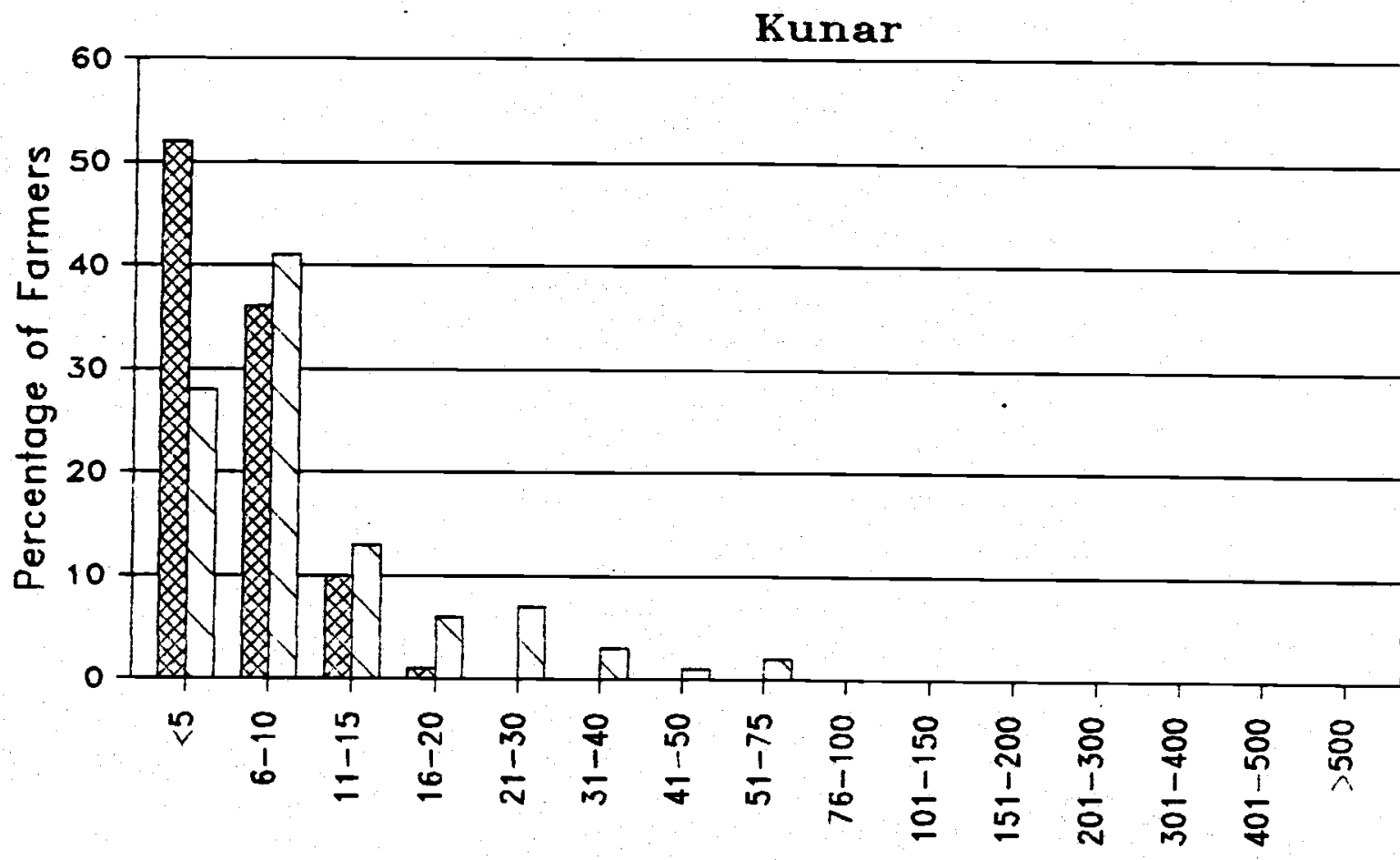

Afghan

$\nabla$ Comps

Range (Jeribs)

1987 Farm Size Distribution Kunar

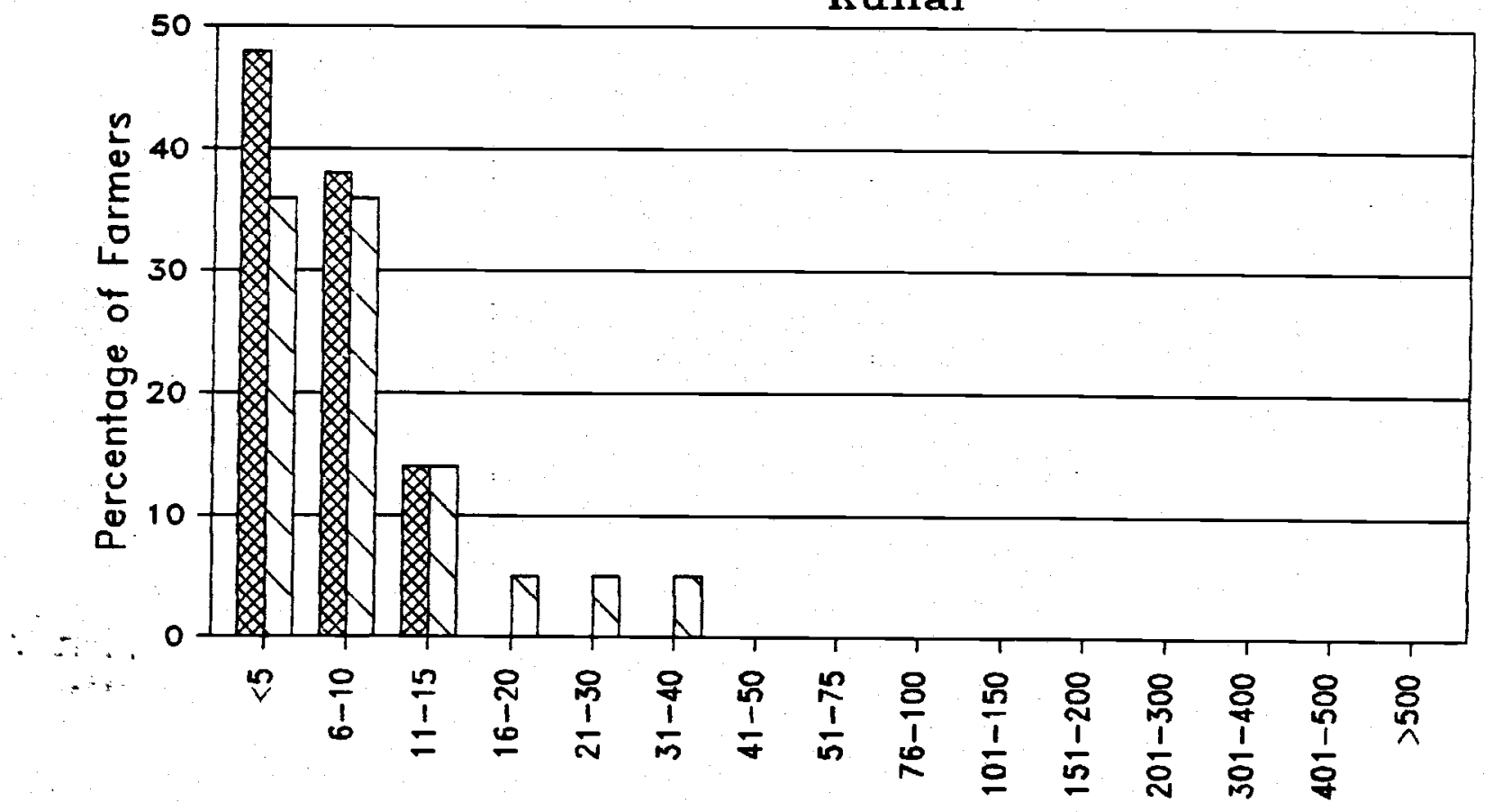

Afghan

$\searrow$ Camps

Range (Jeribs) 
PROVINCE KUNAR

$\begin{array}{lllll}1987 & 1986 & 1985 & 1980 & 1978\end{array}$

Average Area Farmed (excluding

farmers who grew no crops:

$\begin{array}{lllll}6.26 & 6.19 & 6.19 & 6.29 & 5.99\end{array}$

Average Area Irrigated:

$\begin{array}{lllll}5.28 & 5.19 & 5.53 & 5.63 & 4.55\end{array}$

Av̀erage Area Dry Cropped:

0.98

1.00

0.65

0.90

1.44

311

311

311

311

311

Number of Farmers

who did not crop at all:

8

4

30

70

4

TABLE 2B - AVERAGE AREAS FARMED FOR FARMERS IN CAMPS

PROVINCE KUNAR

$\begin{array}{lllll}1987 & 1986 & 1985 & 1980 & 1978\end{array}$

Average. Area Farmed (excluding

farmers who grew no crops:

$\begin{array}{lllll}9.70 & 8.53 & 9.22 & 11.06 & 11.36\end{array}$

Average Area Irrigated:

7.80

7.03

7.35

7.58

7.72

Average Area Dry Cropped:

1.90

1.50

1.75

3.38

3.54

238

238

238

238

238

Number of Farmers

who did not crop at all:

218

72

10

1

0 
NO. FARMS WITH ONLY I. LAND

203.00

66.56

AREA I. - ONLY I. FARMS

1202.00

5.92

3.61

STD I. AREA - ONLY I. FARMS

NO. FARMS WITH ONLY R. LAND

Percent

AREA R. - ONLY R. FARMS

AV. R. AREA/FARM - ONLY R.

STD R. AREA - ONLY R. FARMS

$$
\begin{array}{r}
28.00 \\
9.18 \\
169.00 \\
6.04 \\
3.77 \\
74.00 \\
24.26 \\
182.00 \\
2.46 \\
1.99 \\
273.00 \\
3.69 \\
2.25
\end{array}
$$

NO. FARMS WITH I.\& R. LAND

Percent

STD R. AREA - I\&R FARMS

Kunar

FARMERS SURVEYED IN CAMPS

TOTAL NO FARMS

TOTAL AREA

AVERAGE AREA

STD AREA

NO. FARMS WITH ONLY I. LAND

Percent

AREA I. - ONLY I. FARMS

AV. I. AREA/FARM - ONLY I.

STD I. AREA - ONLY I. FARMS

NO. FARMS WITH ONLY R. LAND

Percent

AREA R. - ONLY R. FARMS

AV . R. AREA/FARM - ONLY R.

STD R. AREA - ONLY R. FARMS

NO. FARMS WITH I.\& R. LAND

Percent

I. AREA FOR I.\& R. FARMS

AV. I - AREA/FARM - I\&R FARMS

STD I .. AREA - I\&R FARMS

R. AREA FOR I.\& R. FARMS

AV. R. AREA/FARM - I\&R FARMS

STD R. AREA -I\&R FARMS

240.00

2717.50

11.32

10.35

138.00

57.50

1176.00

8.52

7.51

3.00

1.25

10.00

3.33

3.30

99.00

41.25

675.50

6.82

5.41

833.00

8.41

8.91 
(For Farmers Afghanistan)

\begin{tabular}{|c|c|c|c|c|c|c|c|c|c|c|c|}
\hline \multirow[t]{2}{*}{ CROP } & \multirow{2}{*}{$\begin{array}{l}\text { Total } \\
\text { for } \\
\text { Average }\end{array}$} & \multicolumn{5}{|c|}{ Numbers who grew crop } & \multicolumn{5}{|c|}{ Percentage who grew Crop } \\
\hline & & 1998 & 1980 & 1985 & 1986 & $198 ?$ & 1978 & 1980 & 1985 & 1986 & 1989 \\
\hline MAIZE & 298 & 265 & 195 & 247 & 264 & 263 & 88.9 & 65.4 & 82.9 & 88.6 & 88.3 \\
\hline IRR WHEAT & 298 & 213 & 197 & 204 & 211 & 210 & 11.5 & 66.1 & 68.5 & 70.8 & 70.5 \\
\hline BARLEY & 298 & 183 & 131 & 141 & 175 & 171 & 61.4 & 44.0 & 47.3 & $58 . ?$ & 59.4 \\
\hline RICE & 298 & 49 & 48 & 49 & 48 & 48 & 16.4 & 16.1 & 15.8 & 16.1 & 16.1 \\
\hline DRY WHEAT & 298 & 20 & 13 & 12 & 17 & 16 & 6.9 & 4,4 & 4.0 & $5 . ?$ & 5.4 \\
\hline BEAN & 298 & 5 & 2 & 2 & 5 & 5 & 1.9 & 0.7 & 0.7 & 1.7 & 1.7 \\
\hline
\end{tabular}




\section{AVERAGE AREA AND YIELD OF CROPS GROWN}

(For Farmers in Afghanistan)

\begin{tabular}{|c|c|c|c|c|c|c|c|c|c|c|c|c|c|}
\hline \multirow[t]{2}{*}{ CROP } & \multirow{2}{*}{$\begin{array}{l}\text { Total } \\
\text { for } \\
\text { Average }\end{array}$} & \multirow{2}{*}{$\begin{array}{l}\text { No.of } \\
\text { Farmers } \\
\text { who grew } \\
\text { Crop (1) }\end{array}$} & \multirow{2}{*}{$\begin{array}{c}\% \text { of } \\
\text { Farmers } \\
\text { who grew } \\
\text { Crop }\end{array}$} & \multicolumn{5}{|c|}{ AVERAGE AREA } & \multicolumn{5}{|c|}{ AVERAGE YIELD } \\
\hline & & & & 1978 & 1980 & 1985 & 1986 & 1987 & 1998 & 1780 & 1985 & 1986 & 1987 \\
\hline IZE & 298 & 265 & 88.9 & 3.5 & 4.2 & 4.1 & 4.0 & 4.0 & 86.9 & 79.9 & 56.1 & 60.2 & 59.5 \\
\hline IRR WHEAT & 298 & 213 & 71.5 & 3.9 & 4.2 & 4.6 & 4.5 & 4. & 80.2 & 73.9 & 51.4 & 56.0 & 56.5 \\
\hline BARLEY & 298 & 183 & 61.4 & 2.9 & 2.6 & 2.6 & 2.8 & 2.7 & 58.9 & 60.5 & 40.4 & 42.3 & 44.3 \\
\hline RICE & 298 & 49 & 16.4 & 2.9 & 2.9 & 3.5 & 3.5 & 3.4 & 90.9 & 84.8 & 67.6 & 59.8 & 59.6 \\
\hline DRY WHEAT & 298 & 20 & 6.7 & 4.2 & 3.7 & 3.4 & 2.5 & 2.4 & 39.1 & 30.4 & 12.8 & 31.5 & 28.3 \\
\hline EAN & 298 & 5 & 1.7 & 1.2 & 2.0 & 1.5 & 1.2 & 1.4 & 45.0 & 40.0 & 35.0 & 30.0 & 25.7 \\
\hline
\end{tabular}

(1) In 1978 
(For Farmers in Camps)

\begin{tabular}{|c|c|c|c|c|c|c|c|c|c|c|c|}
\hline \multirow[t]{2}{*}{ CROP } & \multirow{2}{*}{$\begin{array}{l}\text { Total } \\
\text { for } \\
\text { Average }\end{array}$} & \multicolumn{4}{|c|}{ Numbers who grew crop } & \multicolumn{6}{|c|}{ Percentage who grew Crop } \\
\hline & & 1978 & 1980 & 1985 & 1986 & 1987 & 1998 & 1780 & 1985 & 1986 & 1987 \\
\hline IRR WHEAT & 238 & 234 & 232 & 224 & 161 & 18 & 98.3 & 97.5 & 94.1 & 67.6 & 7.6 \\
\hline MAIZE & 238 & 225 & 223 & 209 & 154 & 2 & 94.5 & 93.7 & 87.8 & 64.7 & 0.8 \\
\hline BARLEY & 238 & 72 & 70 & 59 & 40 & 8 & 30.3 & 29.4 & 24.8 & 16.8 & 3.4 \\
\hline DRY WHEAT & 238 & 64 & 61 & 30 & 26 & 3 & 26.9 & 25.6 & 12.6 & 10.9 & 1.3 \\
\hline RICE & 238 & 63 & 63 & 60 & 39 & 1 & 26.5 & 26.5 & 25.2 & 16.4 & 0.4 \\
\hline BEAN & 238 & 7 & $?$ & 7 & 6 & 0 & 2.9 & 2.9 & 2.9 & 2.5 & 0.0 \\
\hline LENTIL & 238 & 1 & 1 & 1 & 1 . & 0 & 0.4 & 0.4 & 0.4 & 0.4 & 0.0 \\
\hline Compon & 238 & 1 & 1 & 1 & 1 & 0 & 0.4 & 0.1 & 0.4 & 0.4 & 0.0 \\
\hline CLOVER & 238 & 1 & 1 & 1 & 1 & 0 & 0.4 & 0.4 & 0.4 & 0.4 & 0.0 \\
\hline
\end{tabular}




\section{AVERAGE AREA AND YIELD OF CROPS GROWN}

(For Farmers in Camps)

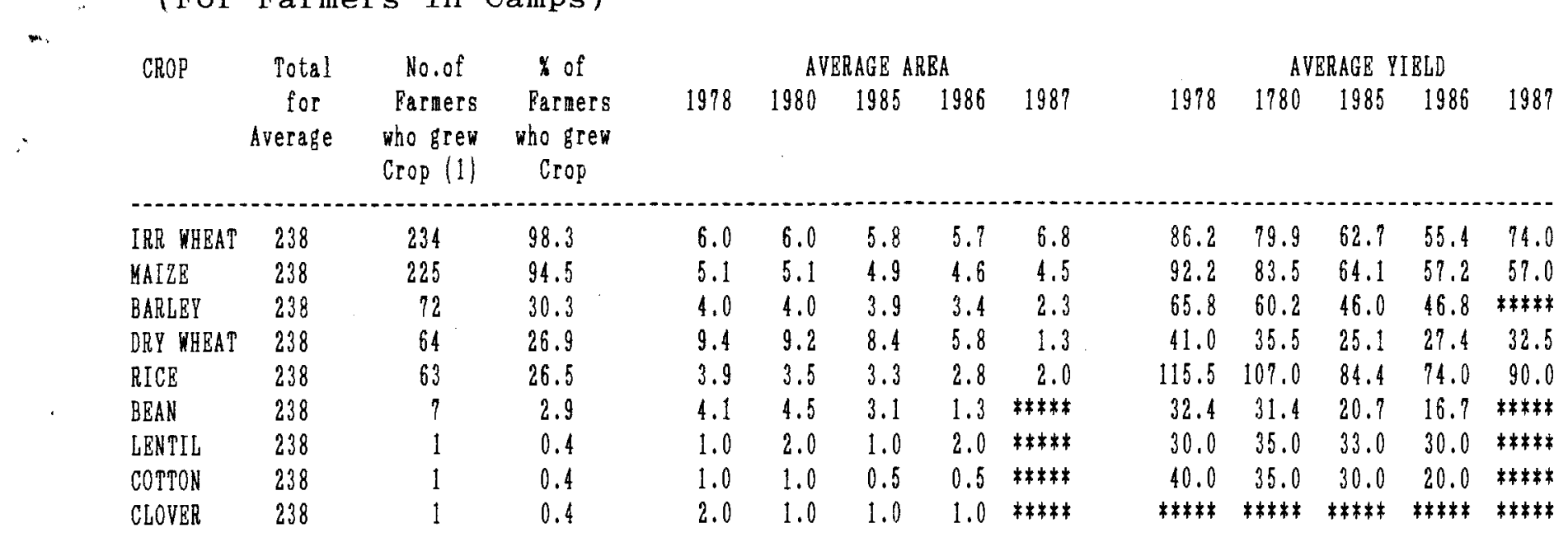

(1) In 1978
PROVINCE: KUNAR 
AVERAGE AREA AND YIELD OF CROPS GROWN

(For both Farmers in Camps and Afghanistan)$$
\text { crop }
$$

CROP

Potal
for

No.of \% of

Farmers

Average who grew who grew

Crop (1) Crop

$\begin{array}{lrrrrrrrr}\text { MAIZE } & 548 & 502 & 91.6 & 4.2 & 4.7 & 4.5 & 4.2 & 4.1 \\ \text { IRR WHEAT } & 548 & 458 & 83.6 & 5.0 & 5.2 & 5.2 & 5.0 & 4.8 \\ \text { BARLEY } & 548 & 262 & 49.8 & 3.2 & 3.1 & 2.9 & 2.9 & 2.9 \\ \text { RICE } & 548 & 114 & 20.8 & 3.4 & 3.2 & 3.3 & 3.2 & 3.3 \\ \text { DRY WHEAT } & 548 & 84 & 15.3 & 8.1 & 8.2 & 7.0 & 4.5 & 2.3 \\ \text { BEAN } & 548 & 13 & 2.4 & 2.7 & 3.9 & 2.8 & 1.3 & 1.9 \\ \text { LENTIL } & 548 & 1 & 0.2 & 1.0 & 2.0 & 1.0 & 2.0 & * * * * * \\ \text { COTTON } & 548 & 1 & 0.2 & 1.0 & 1.0 & 0.5 & 0.5 & * * * * * \\ \text { CLOVBR } & 548 & 1 & 0.2 & 2.0 & 1.0 & 1.0 & 1.0 & * * * *\end{array}$

AVERAGB AREA

PROVINCE: KUNAR

AVERAGE YIELD

$\begin{array}{lllll}1978 & 1980 & 1985 & 1986 & 1987\end{array}$

$\begin{array}{lllll}1998 & 1980 & 1985 & 1986 & 1987\end{array}$

$1978-1980-19851980-1989$

(1) In 1998 
(For both Farmers in Camps and Afghanistan)

\begin{tabular}{|c|c|c|c|c|c|c|c|c|c|c|c|}
\hline \multirow[t]{3}{*}{ CROP } & \multirow{3}{*}{$\begin{array}{l}\text { Total } \\
\text { for } \\
\text { Average }\end{array}$} & \multicolumn{5}{|c|}{ Numbers who grew crop } & \multicolumn{5}{|c|}{ Percentage who grew Crop } \\
\hline & & 1978 & 1980 & 1985 & 1986 & 1989 & 1998 & 1780 & 1985 & 1986 & 1989 \\
\hline & & & & & & & & & & & \\
\hline MAIZE & 548 & 502 & 430 & 468 & 430 & 277 & 91.6 & 78.5 & 85.4 & 78.5 & 50.5 \\
\hline IRR NHEAT & 548 & 458 & 440 & 439 & 383 & 239 & 83.6 & 80.3 & 80.1 & 69.9 & 43.6 \\
\hline BARLEY & 548 & 262 & 208 & $20 ?$ & 222 & 186 & 47.8 & 38.0 & 37.8 & 40.5 & 33.9 \\
\hline RICE & 548 & 114 & 113 & 109 & 89 & 51 & 20.8 & 20.6 & 19.9 & 16.2 & 9.3 \\
\hline DRY WHEAT & 548 & 84 & 74 & 42 & 43 & 19 & 15.3 & 13.5 & 7.7 & 9.8 & 3.5 \\
\hline BEAN & 548 & 13 & 10 & 10 & 12 & 6 & 2.4 & 1.8 & 1.8 & 2.2 & 1.1 \\
\hline LENTIL & 548 & 1 & 1 & 1 & 1 & 0 & 0.2 & 0.2 & 0.2 & 0.2 & 0.0 \\
\hline COTTON & 548 & 1 & 1 & 1 & 1 & 0 & 0.2 & 0.2 & 0.2 & 0.2 & 0.0 \\
\hline CLOVER & 548 & 1 & 1 & 1 & 1 & 0 & 0.2 & 0.2 & 0.2 & 0.2 & 0.0 \\
\hline
\end{tabular}




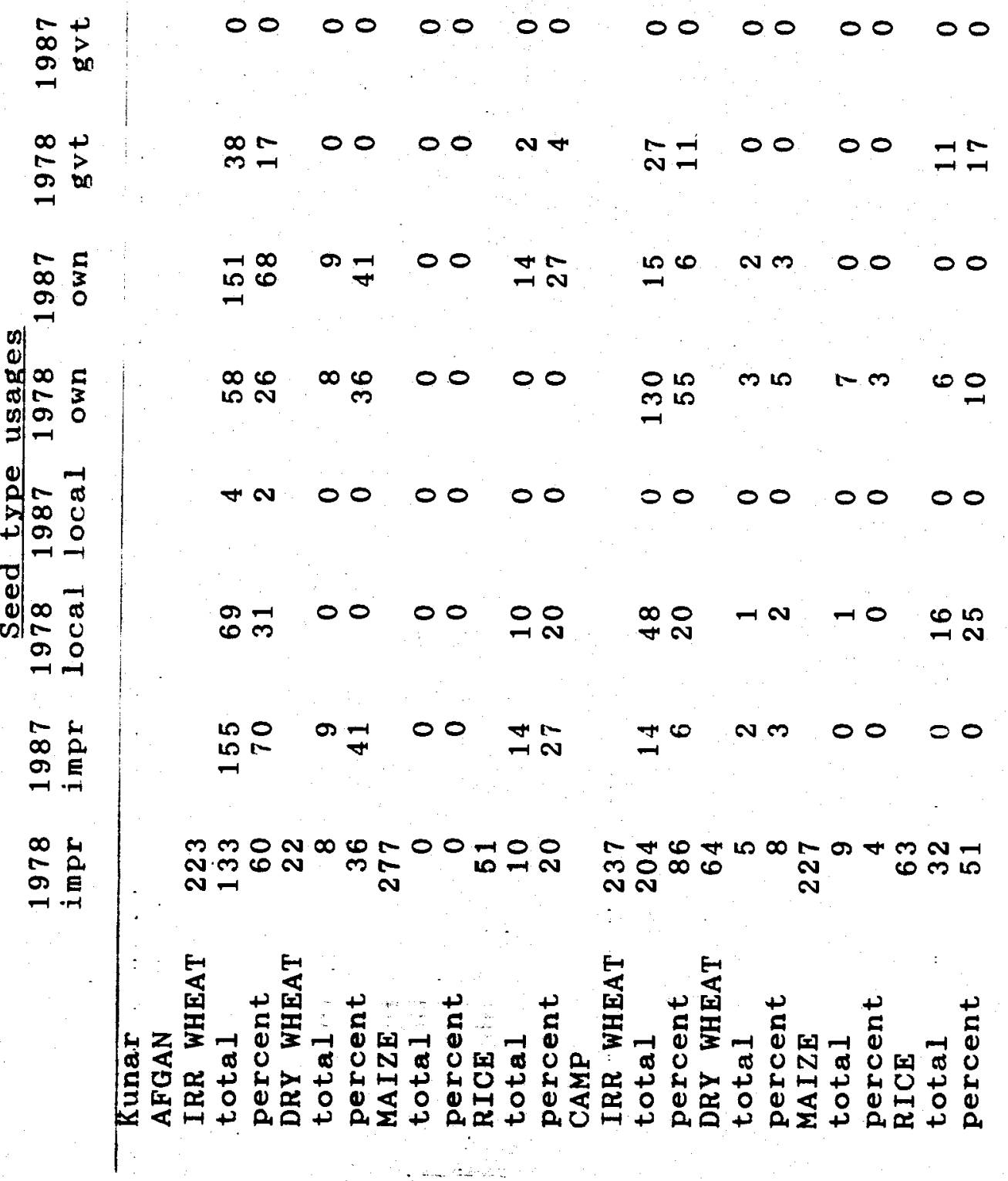


TABLE 4A - METHOD OF LAND PREPARATION - FARMERS IN AFGHANISTAN

PROVINCE KUNAR

PERCENTAGE OF FARMERS WHO USED OWN OXEN

$\begin{array}{lllll}1987 & 1986 & 1985 & 1980 & 1978\end{array}$

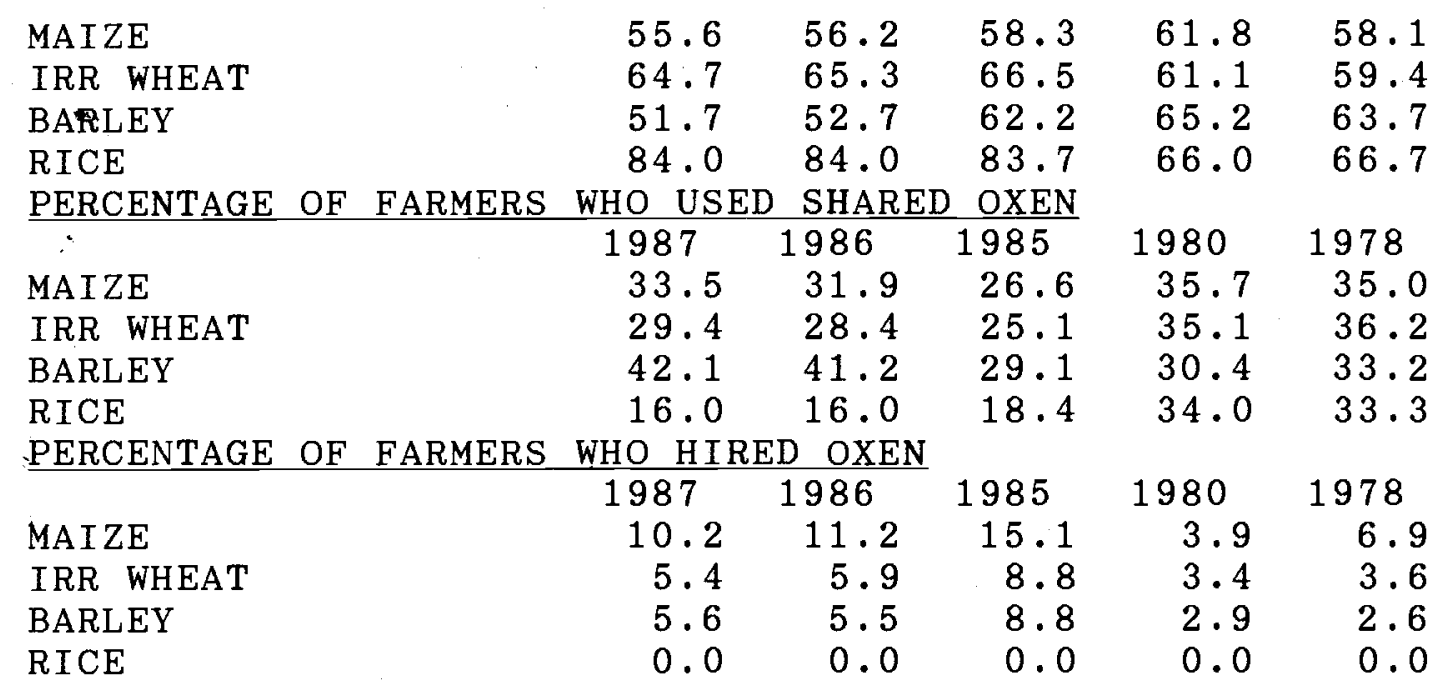

PERCENTAGE OF FARMERS WHO USED OWN TRACTOR

MAIZE

IRR WHEAT

$1987 \quad 1986 \quad 1985$

$1980 \quad 1978$

$\begin{array}{lllll}0.0 & 0.0 & 0.0 & 0.0 & 0.0 \\ 0.0 & 0.0 & 0.0 & 0.0 & 0.0 \\ 0.0 & 0.0 & 0.0 & 0.0 & 0.0 \\ 0.0 & 0.0 & 0.0 & 0.0 & 0.0\end{array}$

RICE

$0.0 \quad 0.0$

0.0

0.0

PERCENTAGE OF FARMERS WHO USED HIRED TRACTORS

\begin{tabular}{lrrrrr} 
& 1987 & 1986 & \multicolumn{1}{c}{1985} & \multicolumn{1}{c}{1980} & 1978 \\
MAIZE & 0.0 & 0.0 & 0.0 & 0.0 & 0.0 \\
IRR WHEAT & 0.0 & 0.0 & 0.0 & 0.0 & 0.0 \\
BARLEY & 0.0 & 0.0 & 0.0 & 0.0 & 0.0 \\
RICE & 0.0 & 0.0 & 0.0 & 0.0 & 0.0
\end{tabular}

PERCENTAGE OF FARMERS WHO USED MANUAL MEANS

MAIZE

$\begin{array}{ccc}1987 & 1986 & 1985 \\ 0.4 & 0.4 & 0.4\end{array}$

$1980 \quad 1978$

IRR WHEAT

$0.0 \quad 0.0 \quad 0.0$

0.0

0.0

BARLEY

0.0

0.0

0.0

0.0

0.0

RICE

0.0

0.0

0.0

0.0

0.0

$0.0 \quad 0.0$

PERCENTAGE OF FARMERS WHO USED OTHER MEANS

\begin{tabular}{lrrrrr} 
& \multicolumn{1}{c}{1987} & 1986 & 1985 & 1980 & 1978 \\
MAIZE & 0.4 & 0.4 & 0.4 & 0.5 & 0.4 \\
IRR WHEAT & 0.5 & 0.5 & 0.5 & 0.5 & 0.4 \\
BARLEY & 0.6 & 0.5 & 0.7 & 0.7 & 0.5 \\
RIGE $\therefore .$. & 0.0 & 0.0 & 0.0 & 0.0 & 0.0 \\
NUMBER OF FARMERS & WHO GREW CROP & & & \\
MAIZE & 1987 & 1986 & 1985 & 1980 & 1978 \\
IRR WHEAT & 275 & 276 & 259 & 207 & 277 \\
BARLEY & 221 & 222 & 215 & 208 & 224 \\
RICE & 178 & 182 & 148 & 138 & 190 \\
& 50 & 50 & 49 & 50 & 51
\end{tabular}


TABLE 4B - METHOD OF LAND PREPARATION- FARMERS IN CAMPS

PROVINCE KUNAR

PERCENTAGE OF FARMERS WHO USED OWN OXEN

$\begin{array}{lllll}1987 & 1986 & 1985 & 1980 & 1978\end{array}$

MAIZE

IRR WHEAT

$150.0 \quad 76.0 \quad 78.0$

84.386 .2

BARLEY

$\begin{array}{lll}72.2 & 76.4 & 76.8\end{array}$

$84.5 \quad 86.3$

RICE

$50.0 \quad 77.5 \quad 78.0$

$84.3 \quad 84.7$

PERCENTAGE OF FARMERS WHO USED SHARED OXEN

\begin{tabular}{lrrrrr}
\hline MAIZE & 1987 & 1986 & 1985 & 1980 & \multicolumn{1}{c}{1978} \\
IRR WHEAT & 100.0 & 18.2 & 16.3 & 11.2 & 9.3 \\
BARLEY & 22.2 & 19.9 & 17.4 & 12.1 & 10.3 \\
RICE & 37.5 & 27.5 & 25.4 & 14.3 & 12.5 \\
PICR & 0.0 & 10.3 & 6.7 & 0.0 & 0.0
\end{tabular}

PERCENTAGE OF FARMERS WHO HIRED OXEN

MAIZE

$\begin{array}{lllll}1987 & 1986 & 1985 & 1980 & 1978\end{array}$

IRR WHEAT

$\begin{array}{lllll}0.0 & 4.5 & 4.3 & 3.6 & 3.6 \\ 0.0 & 5.0 & 4.9 & 3.0 & 3.0 \\ 0.0 & 0.0 & 0.0 & 1.4 & 1.4 \\ 0.0 & 2.6 & 5.0 & 1.6 & 1.6\end{array}$

RICE 
TABLE 4E (CONTD.)

NUMBER OF FARMERS REPORTING NON-AVAILABILTY OF GREY FERTILIZER $\begin{array}{lllll}1987 & 1986 \quad 1985 & 1980 \quad 1978\end{array}$

MAIZE IRR WHEAT BARLEY RICE

$\begin{array}{rrrrr}50 & 58 & 63 & 9 & 0 \\ 60 & 71 & 73 & 4 & 1 \\ 14 & 15 & 14 & 3 & 1 \\ 6 & 8 & 9 & 0 & 0\end{array}$

NUMBER OF FARMERS WHO GREW CROP

$\begin{array}{lllll}1987 & 1986 & 1985 & 1980 & 1978\end{array}$

MAI ZE

IRR WHEAT

BARLEY

RICE
263

210

171

48

\section{4}

211

175

48
247

204

141

47
195

197

131

48
265

213

183

49

NUMBER OF FARMERS WHO USED WHITE FERTILIZER

$\begin{array}{lrrrrr} & 1987 & 1986 & 1985 & 1980 & 1978 \\ \text { MAIZE } & 116 & 88 & 44 & 118 & 187 \\ \text { IRR WHEAT } & 95 & 55 & 39 & 127 & 158 \\ \text { BARLEY } & 28 & 14 & 11 & 43 & 56 \\ \text { RICE } & 16 & 6 & 5 & 36 & 38\end{array}$

NUMBER OF FARMERS WHO USED GREY FERTILIZER

$\begin{array}{lllll}1987 & 1986 & 1985 & 1980 & 1978\end{array}$

MAIZE

$\begin{array}{rrrrr}46 & 41 & 26 & 50 & 65 \\ 50 & 40 & 35 & 106 & 117 \\ 3 & 3 & 2 & 1 & 2 \\ 6 & 4 & 3 & 16 & 17\end{array}$

IRR WHEAT

BARLEY

RICE

$\begin{array}{lllll}6 & 4 & 3 & 16 & 17\end{array}$ 
TABLE 4F - FERTILIZER USE, COST AND AVAILABILITY - FARMERS IN CAMPS

\begin{tabular}{llll} 
& \multicolumn{2}{c}{ PROVINCE KUNAR } \\
AVERAGE USE OF & WHITE FERTILIZER \\
\hline
\end{tabular}

MAIZE

$1987 \quad 1986 \quad 1985 \quad 1980 \quad 1978$

IRR WHEAT

$* * * * *$

0.50

0.50

0.50

0.54

0.53

BARLEY

$* * * * *$

0.52

0.54

0.57

0.57

RICE

$* * * * *$

0.50

0.47

0.56

0.57

0.69

0.62

0.62

0.61

AVERAGE PRICE OF WHITE FERTILIZER

$$
\begin{array}{lllll}
1987 & 1986 & 1985 & 1980 & 1978
\end{array}
$$

MATZE

IRR WHEAT

BARLEY

$666 \quad 479$

RICE

$\begin{array}{rrrrr}900 & 1228 & 1014 & 650 & 476 \\ * * * * * & 1244 & 1002 & 634 & 490 \\ * * * * * & 1258 & 1117 & 702 & 458\end{array}$

NUMBER OF FARMERS REPORTING NON-AVAILABILTY OF WHITE FERTILIZER

$$
1987 \quad 1986 \quad 1985 \quad 1980 \quad 1978
$$

$\begin{array}{lrrrrr}\text { MATZE } & 3 & 49 & 50 & 13 & 0 \\ \text { IRR WHEAT } & 15 & 52 & 56 & 13 & 0 \\ \text { BARLEY } & 3 & 8 & 9 & 3 & 0 \\ \text { RICE } & 1 & 22 & 20 & 5 & 0\end{array}$

AVERAGE USE OF GREY FERTILIZER

$\begin{array}{lrrrrr}\text { MATZE } & * * * * * & 0.38 & 0.42 & 0.44 & 0.44 \\ \text { IRR WHEAT } & 0.50 & 0.46 & 0.46 & 0.46 & 0.45 \\ \text { BARLEY } & * * * * * & 0.50 & 0.44 & 0.61 & 0.57 \\ \text { RICE } & * * * * * & 0.40 & 0.42 & 0.42 & 0.41\end{array}$

$\begin{array}{lllll}1987 & 1986 & 1985 & 1980 & 1978\end{array}$

AVERAGE PRICE OF GREY FERTILIZER

$\begin{array}{lllll}1987 & 1986 & 1985 & 1980 & 1978\end{array}$

MATZE

IRR WHEAT

BARLEY

RICE

$\begin{array}{rrrrr}* * * * * & 1196 & 988 & 730 & 568 \\ 1050 & 1177 & 967 & 709 & 575 \\ * * * * * & 1283 & 903 & 705 & 573 \\ * * * * * & 1256 & 1160 & 788 & 560\end{array}$


TABLE $4 \mathrm{~F}$ (CONTD.)

NUMBER OF FARMERS REPORTING NON-AVAILABILTY OF GREY FERTILIZER $\begin{array}{lllll}1987 & 1986 & 1985 & 1980 & 1978\end{array}$

MÄIZE

IRR WHEAT

BARLEY

RİCE

$\begin{array}{rrrrr}2 & 28 & 32 & 8 & 1 \\ 9 & 39 & 45 & 13 & 0 \\ 1 & 4 & 7 & 4 & 0 \\ 0 & 12 & 8 & 4 & 0\end{array}$

NUMBER OF FARMERS WHO GREW CROP

$\begin{array}{lllll}1987 & 1986 & 1985 & 1980 & 1978\end{array}$

MAIZE

IRR WHEAT

BARLEY

RICE

$\begin{array}{rrrrr}2 & 154 & 209 & 223 & 225 \\ 18 & 161 & 224 & 232 & 234 \\ 8 & 40 & 59 & 70 & 72 \\ 1 & 39 & 60 & 63 & 63\end{array}$

NUMBER OF FARMERS WHO USED WHITE FERTILIZER

$\begin{array}{lllll}1987 & 1986 & 1985 & 1980 & 1978\end{array}$

$\begin{array}{lrrrrr}\text { MAIZE } & 0 & 53 & 99 & 156 & 184 \\ \text { IRR WHEAT } & 1 & 65 & 120 & 177 & 201 \\ \text { BARLEY } & 0 & 8 & 14 & 25 & 30 \\ \text { RICE } & 0 & 13 & 30 & 52 & 58\end{array}$

NUMBER OF FARMERS WHO USED GREY FERTILIZER

$\begin{array}{lllll}1987 & 1986 & 1985 & 1980 & 1978\end{array}$

MAIZE

IRR WHEAT

BARLEY

RICE

$\begin{array}{rrrrr}0 & 32 & 56 & 98 & 117 \\ 1 & 43 & 87 & 147 & 166 \\ 0 & 3 & 4 & 11 & 15 \\ 0 & 9 & 19 & 33 & 39\end{array}$


TABLE 7A(1) - AVERAGE NUMBERS OF TRAINED OXEN FOR FARMERS IN AFGANISTAN

PROVINCE KUNAR

Number of Farmers Questioned 298

TABLE 7A(2) - AVERAGE NUMBER OF TRAINED OXEN FOR FARMERS IN THE CAMPS

PROVINCE KUNAR

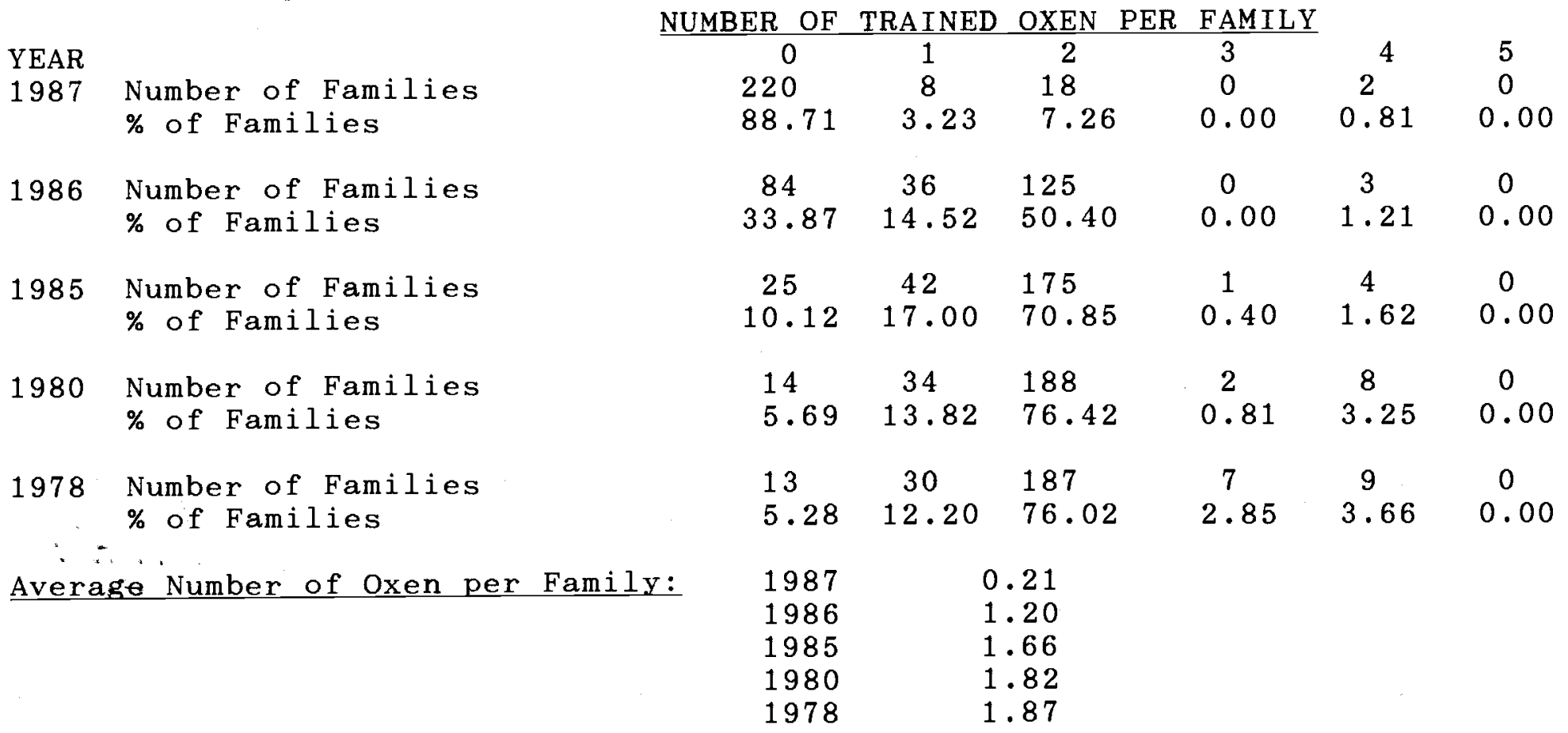

Number of Farmers Questioned 248 
TABLE 7B(1) - AVERAGE PERCENT OF FARMS WITH OTHER ANIMALS FOR FARMERS IN AFGANISTAN

PROVINCE KUNAR

Horses

Doñkeys and Mules

Camels

Karakul Sheep

Other Sheep and Goats

$1987 \quad 1986$

1985

$1980 \quad 1978$

2.35

48.99

0.00

2.35

2.35

42.28

0.00

2.01

2.35

0.00

0.00

0.00

36.24

0.00

0.00

81.21

79.87

72.48

0.00

44.97

0.00

0.00

$67.79 \quad 89.26$

Number of Farmers Questioned 298

TABLE 7B(2) - AVERAGE PERCENT OF FARMS WITH OTHER ANIMALS FOR FARMERS IN CAMPS

PROVINCE

KUNAR

$1987 \quad 1986 \quad 1985 \quad 1980 \quad 1978$

Horses

Donkeys and Mules

Camels

Karakul sheep

Other Sheep and Goats

1.61

7.66

0.00

0.00

8.47

2.02

4.03

26.61

0.00

45.56

0.00

0.00 .

1.21

40.32

69.35

4.44

50.40

0.00

1.61

82.66
4.44

47.58

0.00

1.61

84.27

Number of Farmers Questioned 248 
TABLE 7B(3) - AVERAGE NO OF ANIMALS (on farms which have them) FOR FARMERS IN AFGANISTAN

PROVINCE KUNAR DISTRICT

$\begin{array}{lrrrrr}\text { Horses } & 1.00 & 1.00 & 1.14 & 1.17 & 1.00 \\ \text { Donkeys and Mules } & 1.23 & 1.23 & 1.19 & 1.21 & 1.26 \\ \text { Camels } & * * * * * & * * * * * & * * * * * & * * * * * & * * * * * \\ \text { Karakul Sheep } & * * * * * & * * * * * & * * * * * & * * * * * & * * * * * \\ \text { Other Sheep and Goats } & 33.88 & 31.55 & 41.81 & 73.35 & 89.94\end{array}$

TABLE 7B(4) - AVERAGE NO OF ANIMALS (on farms which have them) FOR FARMERS IN CAMPS PROVINCE KUNAR

$\begin{array}{lrrrrr}\text { Horses } & 1.50 & 1.40 & 1.40 & 1.64 & 1.64 \\ \text { Donkeys and Mules } & 1.47 & 1.53 & 1.51 & 1.54 & 1.62 \\ \text { Camels } & * * * * * & * * * * * & * * * * * & * * * * * & * * * * * \\ \text { Karakul Sheep } & * * * * * & * * * * * & 28.33 & 53.75 & 60.00 \\ \text { Other Sheep and Goats } & 36.90 & 32.90 & 38.20 & 48.79 & 47.82\end{array}$


TABLE 7B(1) - AVERAGE PERCENT OF FARMS WITH OTHER ANIMALS FOR FARMERS IN AFGANISTAN

PROVINCE

KUNAR

$1987 \quad 1986 \quad 1985 \quad 1980 \quad 1978$

$\begin{array}{lrrrrr}\text { Horses } & 2.35 & 2.35 & 2.35 & 2.01 & 2.35 \\ \text { Donkeys and Mules } & 48.99 & 48.66 & 42.28 & 36.24 & 44.97 \\ \text { Camels } & 0.00 & 0.00 & 0.00 & 0.00 & 0.00 \\ \text { Karakul Sheep } & 0.00 & 0.00 & 0.00 & 0.00 & 0.00 \\ \text { Q.ther Sheep and Goats } & 81.21 & 79.87 & 72.48 & 67.79 & 89.26\end{array}$

Number of Farmers Questioned 298

TABLE 7B(2) - AVERAGE PERCENT OF FARMS WITH OTHER ANIMALS FOR FARMERS IN CAMPS

PROVINCE KUNAR DISTRICT

$$
1987 \quad 1986 \quad 1985 \quad 1980 \quad 1978
$$

$\begin{array}{lrrrrr}\text { Horses } & 1.61 & 2.02 & 4.03 & 4.44 & 4.44 \\ \text { Donkeys and Mules } & 7.66 & 26.61 & 45.56 & 50.40 & 47.58 \\ \text { Camels } & 0.00 & 0.00 & 0.00 & 0.00 & 0.00 \\ \text { Karakul Sheep } & 0.00 & 0.00 & 1.21 & 1.61 & 1.61 \\ \text { Other Sheep and Goats } & 8.47 & 40.32 & 69.35 & 82.66 & 84.27\end{array}$

Number of Farmers Questioned 248 
TABLE 7B(3) - AVERAGE NO OF ANIMALS (on farms which have them) FOR FARMERS IN AFGANISTAN

PROVINCE KUNAR DISTRICT

$\begin{array}{lrrrrr} & 1987 & 1986 & 1985 & 1980 & 1978 \\ \text { Horses } & 1.00 & 1.00 & 1.14 & 1.17 & 1.00 \\ \text { Denkeys and Mules } & 1.23 & 1.23 & 1.19 & 1.21 & 1.26 \\ \text { Camels } & * * * * * & * * * * * & * * * * * & * * * * * & * * * * * \\ \text { Karakul Sheep } & * * * * * & * * * * * & * * * * * & * * * * * & * * * * * \\ \text { Other Sheep and Goats } & 33.88 & 31.55 & 41.81 & 73.35 & 89.94\end{array}$

TABLE 7B(4) - AVERAGE NO OF ANIMALS (on farms which have them) FOR FARMERS IN CAMPS PROVINCE KUNAR

$\begin{array}{lrrrrr} & 1987 & 1986 & 1985 & 1980 & 1978 \\ & & & & & \\ \text { Horses } & 1.50 & 1.40 & 1.40 & 1.64 & 1.64 \\ \text { Donkeys and Mules } & 1.47 & 1.53 & 1.51 & 1.54 & 1.62 \\ \text { Camels } & * * * * * & * * * * * & * * * * * & * * * * * & * * * * * \\ \text { Karakul Sheep } & * * * * * & * * * * * & 28.33 & 53.75 & 60.00 \\ \text { Other Sheep and Goats } & 36.90 & 32.90 & 38.20 & 48.79 & 47.82\end{array}$


TABLE 7C(1) - AVERAGE SIZE OF CATTLE HERD FOR FARMERS IN AFGANISTAN

PROVINCE KUNAR

\begin{tabular}{|c|c|c|c|c|c|c|c|c|c|c|}
\hline \multicolumn{11}{|c|}{ SIZE OF HERD } \\
\hline YEAR & & 0 & 1 & 2 & 3 & 4 & 5 & 6 & 7 & Over 7 \\
\hline 1987 & $\begin{array}{l}\text { Number of Herds } \\
\% \text { of Herds }\end{array}$ & $\begin{array}{l}30 \\
10.07\end{array}$ & $\begin{array}{c}8 \\
2.68\end{array}$ & $\begin{array}{l}15 \\
5.03\end{array}$ & $\begin{array}{c}33 \\
11.07\end{array}$ & $\begin{array}{c}44 \\
14.77\end{array}$ & $\begin{array}{c}47 \\
15.77\end{array}$ & $\begin{array}{c}31 \\
10.40\end{array}$ & $\begin{array}{l}25 \\
8.39\end{array}$ & $\begin{array}{c}65 \\
2181\end{array}$ \\
\hline & & & & & & & & & & \\
\hline 1986 & $\begin{array}{l}\text { Number of Herds } \\
\% \text { of Herds }\end{array}$ & $\begin{array}{l}28 \\
9.40\end{array}$ & $\begin{array}{l}14 \\
4.70\end{array}$ & $\begin{array}{l}24 \\
8.05\end{array}$ & $\begin{array}{c}33 \\
11.07\end{array}$ & $\begin{array}{c}54 \\
18.12\end{array}$ & $\begin{array}{c}38 \\
12.75\end{array}$ & $\begin{array}{l}25 \\
8.39\end{array}$ & $\begin{array}{l}21 \\
7.05\end{array}$ & $\begin{array}{c}61 \\
20.47\end{array}$ \\
\hline 1985 & $\begin{array}{l}\text { Number of Herds } \\
\% \text { of Herds }\end{array}$ & $\begin{array}{c}63 \\
21.14\end{array}$ & $\begin{array}{l}26 \\
8.72\end{array}$ & $\begin{array}{l}14 \\
4.70\end{array}$ & $\begin{array}{l}25 \\
8.39\end{array}$ & $\begin{array}{c}30 \\
10.07\end{array}$ & $\begin{array}{c}47 \\
15.77\end{array}$ & $\begin{array}{l}28 \\
9.40\end{array}$ & $\begin{array}{l}22 \\
7.38\end{array}$ & $\begin{array}{c}43 \\
14.43\end{array}$ \\
\hline 1980 & $\begin{array}{l}\text { Number of Herds } \\
\% \text { of Herds }\end{array}$ & $\begin{array}{c}73 \\
24.50\end{array}$ & $\begin{array}{c}5 \\
1.68\end{array}$ & $\begin{array}{l}10 \\
3.36\end{array}$ & $\begin{array}{l}10 \\
3.36\end{array}$ & $\begin{array}{c}43 \\
14.43\end{array}$ & $\begin{array}{l}24 \\
8.05\end{array}$ & $\begin{array}{l}25 \\
8.39\end{array}$ & $\begin{array}{c}33 \\
11.07\end{array}$ & $\begin{array}{c}75 \\
25.17\end{array}$ \\
\hline 1978 & $\begin{array}{l}\text { Number of Herds } \\
\% \text { of Herds }\end{array}$ & $\begin{array}{c}4 \\
1.34\end{array}$ & $\begin{array}{c}0 \\
0.00\end{array}$ & $\begin{array}{c}4 \\
1.34\end{array}$ & $\begin{array}{l}21 \\
7.05\end{array}$ & $\begin{array}{l}23 \\
7.72\end{array}$ & $\begin{array}{c}41 \\
13.76\end{array}$ & $\begin{array}{c}40 \\
13.42\end{array}$ & $\begin{array}{c}37 \\
12.42\end{array}$ & $\begin{array}{l}128 \\
42.95\end{array}$ \\
\hline Averag & $\begin{array}{r}1987 \\
1986 \\
1985 \\
1980 \\
1978\end{array}$ & & $\begin{array}{l}5.26 \\
4.87 \\
4.15 \\
. .24 \\
7.58\end{array}$ & & & & & & & \\
\hline
\end{tabular}

Number of Farmers Questioned 298

TABLE 7C(2) - AVERAGE SIZE OF CATTLE HERDS FOR FARMERS IN THE CAMPS PROVINCE KUNAR

\begin{tabular}{|c|c|c|c|c|c|c|c|c|c|c|c|}
\hline \multirow{3}{*}{$\begin{array}{l}\text { YEAR } \\
1987\end{array}$} & \multicolumn{7}{|c|}{ SIZE OF HERD } & \multirow[b]{2}{*}{5} & \multirow[b]{2}{*}{6} & \multirow[b]{2}{*}{7} & \multirow[b]{2}{*}{ Over 7} \\
\hline & & & 0 & 1 & 2 & 3 & 4 & & & & \\
\hline & $\begin{array}{l}\text { Number of Her } \\
\% \text { of Herds }\end{array}$ & erds & $\begin{array}{l}219 \\
88.31\end{array}$ & $\begin{array}{c}0 \\
0.00\end{array}$ & $\begin{array}{c}1 \\
0.40\end{array}$ & $\begin{array}{c}2 \\
0.81\end{array}$ & $\begin{array}{c}4 \\
1.61\end{array}$ & $\begin{array}{c}2 \\
0.81\end{array}$ & $\begin{array}{c}2 \\
0.81\end{array}$ & $\begin{array}{c}4 \\
1.61\end{array}$ & $\begin{array}{l}14 \\
5.65\end{array}$ \\
\hline 1986 & $\begin{array}{l}\text { Number of Her } \\
\% \text { of Herds }\end{array}$ & erds & $\begin{array}{c}73 \\
29.44\end{array}$ & $\begin{array}{c}8 \\
3.23\end{array}$ & $\begin{array}{c}32 \\
12.90\end{array}$ & $\begin{array}{l}20 \\
8.06\end{array}$ & $\begin{array}{c}43 \\
17.34\end{array}$ & $\begin{array}{l}19 \\
7.66\end{array}$ & $\begin{array}{l}18 \\
7.26\end{array}$ & $\begin{array}{l}11 \\
4.44\end{array}$ & $\begin{array}{l}24 \\
9.68\end{array}$ \\
\hline $1985 !$ & $\begin{array}{l}\text { Number of Her } \\
\% \text { of Herds }\end{array}$ & erds & $\begin{array}{l}14 \\
5.65\end{array}$ & $\begin{array}{c}3 \\
1.21\end{array}$ & $\begin{array}{l}14 \\
5.65\end{array}$ & $\begin{array}{l}21 \\
8.47\end{array}$ & $\begin{array}{c}51 \\
20.56\end{array}$ & $\begin{array}{c}34 \\
13.71\end{array}$ & $\begin{array}{c}40 \\
16.13\end{array}$ & $\begin{array}{l}16 \\
6.45\end{array}$ & $\begin{array}{c}55 \\
22.18\end{array}$ \\
\hline $1980 ?$ & $\begin{array}{l}\text { Number of Her } \\
\% \text { of Herds }\end{array}$ & erds & $\begin{array}{c}1 \\
0.40\end{array}$ & $\begin{array}{c}1 \\
0.40\end{array}$ & $\begin{array}{c}5 \\
2.02\end{array}$ & $\begin{array}{c}7 \\
2.82\end{array}$ & $\begin{array}{l}24 \\
9.68\end{array}$ & $\begin{array}{c}28 \\
11.29\end{array}$ & $\begin{array}{l}24 \\
9.68\end{array}$ & $\begin{array}{c}35 \\
14.11\end{array}$ & $\begin{array}{l}123 \\
49.60\end{array}$ \\
\hline $1978 \frac{1}{9}$ & $\begin{array}{l}\text { Number of Her } \\
\% \text { of Herds }\end{array}$ & erds & $\begin{array}{c}0 \\
0.00\end{array}$ & $\begin{array}{c}0 \\
0.00\end{array}$ & $\begin{array}{c}3 \\
1.21\end{array}$ & $\begin{array}{c}9 \\
3.63\end{array}$ & $\begin{array}{l}22 \\
8.87\end{array}$ & $\begin{array}{l}19 \\
7.66\end{array}$ & $\begin{array}{c}48 \\
19.35\end{array}$ & $\begin{array}{c}25 \\
10.08\end{array}$ & $\begin{array}{l}122 \\
49.19\end{array}$ \\
\hline erage & se Herd Size $\begin{array}{r}1 \\
1 \\
1 \\
1\end{array}$ & $\begin{array}{l}1987 \\
1986 \\
1985 \\
1980 \\
1978\end{array}$ & & $\begin{array}{l}0.85 \\
3.41 \\
5.68 \\
8.46 \\
8.58\end{array}$ & & & & & & & \\
\hline
\end{tabular}


TABLE 8(A) - AVERAGE LABOUR (FAMILY and HIRED) FOR FARMERS IN AFGANISTAN PROVINCE KUNAR

\begin{tabular}{|c|c|c|c|c|c|}
\hline $\begin{array}{l}\text { Average Family Labour per Farm } \\
\text {. }\end{array}$ & 1.40 & 1.41 & 1.28 & 1.25 & 1.73 \\
\hline $\begin{array}{l}\text { Average No. of Hired Labour per Farm } \\
\text { (for farms using hired labour) }\end{array}$ & 1.00 & 1.00 & $* * * * *$ & $* * * * *$ & 1.00 \\
\hline Number of Farms using Hired Labour & 2 & 2 & 0 & 0 & 1 \\
\hline
\end{tabular}

TABLE 8(B) - AVERAGE LABOUR (FAMILY and HIRED) FOR FARMERS IN CAMPS

PROVINCE KUNAR

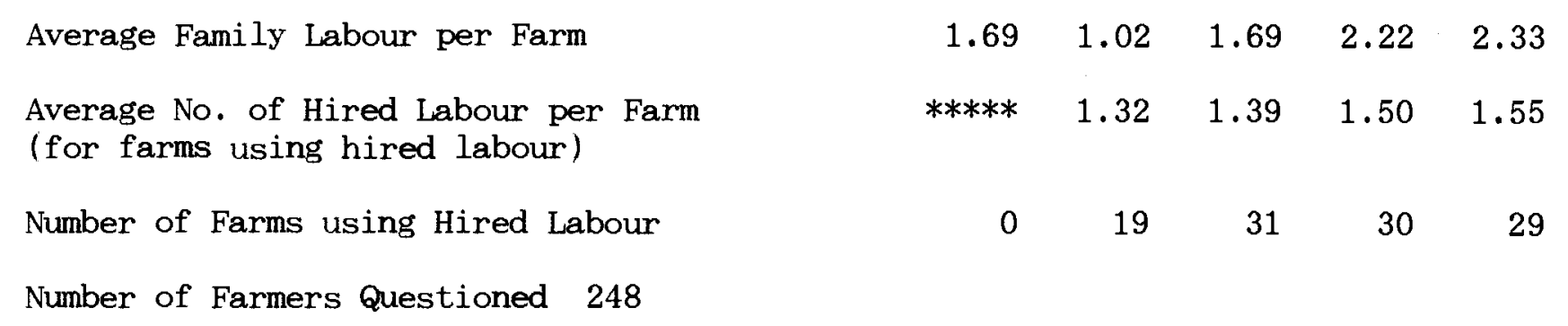


TABLE 9A - DIRECT EFFECTS OF THEWAR FARMFRS IN AFGANISTAN

PROVINCE OF : KUNAR

$\begin{array}{lllll}1987 & 1986 \quad 1985 & 1980 & 1978\end{array}$

Destruction of Irrigation System

Nos of Farmers

Percent of Farmers

$\begin{array}{rrrrr}28 & 52 & 54 & 29 & 3 \\ 9.03 & 16.77 & 17.42 & 9.35 & 0.97\end{array}$

Burning of Crops

Nòs of Farmers

Percent of Farmers

$\begin{array}{rrrrr}4 & 15 & 37 & 16 & 2 \\ 1.29 & 4.84 & 11.94 & 5.16 & 0.65\end{array}$

Bombing of Villages

Nos of Farmers

$\begin{array}{rrrrr}53 & 117 & 186 & 151 & 7 \\ 17.10 & 37.74 & 60.00 & 48.71 & 2.26\end{array}$

Destruction of Grainstores

Nos of Farmers

$\begin{array}{rrrrr}5 & 20 & 34 & 15 & 1 \\ 1.61 & 6.45 & 10.97 & 4.84 & 0.32\end{array}$

Other

Nos of Farmers

Percent of Farmers

$\begin{array}{rrrrr}0 & 1 & 0 & 1 & 0 \\ 0.00 & 0.32 & 0.00 & 0.32 & 0.00\end{array}$

Average Number of Livestock Shot

$\begin{array}{lllll}2.54 & 3.40 & 7.27 & 3.57 & 30.00\end{array}$

Number of Farmers reporting

Livestock Shot

$\begin{array}{lllll}13.00 & 20.00 & 64.00 & 35.00 & 2.00\end{array}$

Average Number of Livestock Killed

by Mines

$\begin{array}{lllll}4.00 & 5.78 & 4.06 & 4.94 & 3.33\end{array}$

Number of Farmers reporting Livestock

killed by Mines

$\begin{array}{lllll}1.00 & 9.00 & 17.00 & 17.00 & 3.00\end{array}$

Number of Farmers Questioned 310 
TABLE 9B - DIRECT EFFECTS OF THE WAR FARMERS IN CAMPS

PROVINCE OF: KUNAR

$\begin{array}{lllll}1987 & 1986 & 1985 & 1980 & 1978\end{array}$

Destruction of Irrigation System

Nas of Farmers

Percent of Farmers

Burning of Crops

Nos of Farmers

Percent of Farmers

Bombing of Villages

Nos of Farmers

Percent of Farmers

Destruction of Grainstores

Nos of Farmers

Percent of Farmers

Other

Nos of Farmers

Percent of Farmers

Average Number of Livestock Shot

Number of Farmers reporting

Livestock Shot

Average Number of Livestock Killed

by Mines

Number of Farmers reporting Livestock killed by Mines

0.00
19

$8.05 \quad 0.00$

$0.00 \quad 11.44 \quad 21.61$

$\begin{array}{rrrrr}0 & 15 & 36 & 9 & 0 \\ 0.00 & 6.36 & 15.25 & 3.81 & 0.00\end{array}$

$\begin{array}{rrrrr}0 & 82 & 166 & 81 & 1 \\ 0.00 & 34.75 & 70.34 & 34.32 & 0.42\end{array}$

$\begin{array}{rrrrr}0 & 9 & 32 & 11 & 0 \\ 0.00 & 3.81 & 13.56 & 4.66 & 0.00\end{array}$

$\begin{array}{rrrrr}0 & 0 & 0 & 0 & 0 \\ 0.00 & 0.00 & 0.00 & 0.00 & 0.00\end{array}$

$\begin{array}{lllll}* * * * * & 22.05 & 11.55 & 10.43 & * * * * *\end{array}$

$\begin{array}{lllll}0.00 & 21.00 & 78.00 & 23.00 & 0.00\end{array}$

***** $\quad 3.83 \quad 8.94 \quad 10.18 \quad * * * * *$

Number of Farmers Questioned 236 
IABLE 1-5 - ANALYSIS OF GREATEST FARMING PROBLEMS - COMMUNITY SURVEY

PROVINCE OF KNR

1987 PERCENTAGE OF VILLAGES GIVING FOLLONING PRIORITY TO PROBLEM

Rainfall

Crop Disease

Birds or Rats

Seed Availability

Fertilizer Availability

Crop Protection Chenicals

Credit

Irrigation Water Avail ability

Flooding

Labour Availabilty

Power for Land Preparation

Direct War Effects

Animal Diseases

Extension

Other Problems

\begin{tabular}{rrrrrrrrrrrrr}
1 & 2 & 3 & 4 & 5 & 6 & 7 & 8 & 9 & 10 & 11 & 12 & Weighted Priority \\
\hline 0 & 0 & 8 & 6 & 2 & 0 & 2 & 0 & 0 & 0 & 0 & 0 & 2.05 \\
3 & 19 & 16 & 13 & 6 & 2 & 3 & 2 & 0 & 0 & 0 & 0 & 10.02 \\
0 & 3 & 27 & 16 & 13 & 6 & 0 & 0 & 0 & 0 & 0 & 0 & 7.79 \\
0 & 5 & 6 & 8 & 13 & 11 & 5 & 5 & 2 & 0 & 0 & 0 & 5.29 \\
16 & 15 & 2 & 2 & 0 & 0 & 0 & 0 & 0 & 0 & 0 & 0 & 10.27 \\
0 & 0 & 2 & 0 & 6 & 15 & 10 & 0 & 0 & 0 & 0 & 0 & 2.38 \\
0 & 5 & 0 & 0 & 3 & 2 & 2 & 0 & 0 & 0 & 0 & 0 & 1.51 \\
11 & 15 & 10 & 3 & 3 & 0 & 0 & 0 & 0 & 0 & 0 & 0 & 9.81 \\
0 & 2 & 3 & 3 & 3 & 0 & 0 & 0 & 0 & 0 & 0 & 0 & 1.41 \\
0 & 0 & 5 & 8 & 8 & 8 & 0 & 0 & 0 & 0 & 0 & 0 & 2.78 \\
2 & 5 & 0 & 5 & 2 & 2 & 0 & 0 & 0 & 0 & 0 & 0 & 2.46 \\
58 & 3 & 6 & 11 & 13 & 3 & 0 & 0 & 0 & 0 & 0 & 0 & 28.62 \\
0 & 3 & 3 & 5 & 3 & 6 & 6 & 0 & 2 & 0 & 0 & 0 & 2.84 \\
0 & 2 & 3 & 8 & 0 & 2 & 0 & 0 & 0 & 0 & 0 & 0 & 1.76 \\
8 & 23 & 8 & 6 & 10 & 2 & 2 & 0 & 0 & 0 & 0 & 0 & 11.02
\end{tabular}

1986

Rainfall

Crop Disease

Birds or Rats

Seed Availability

Fertilizer Availability

Crop Protection Chemicals

Credit

Irrigation Water Availability

flooding

Labour Availabilty

Power for Land Preparation

Direct War Effects

Animal Diseases

Extension

Other Problems

\begin{tabular}{rrrrrrrrrrrrr}
1 & 2 & 3 & 4 & 5 & 6 & 7 & 8 & 9 & 10 & 11 & 12 & Weighted Priority \\
\hline 0 & 2 & 5 & 3 & 3 & 0 & 5 & 2 & 0 & 0 & 0 & 0 & 2.00 \\
0 & 23 & 18 & 11 & 10 & 5 & 0 & 0 & 0 & 0 & 0 & 0 & 9.55 \\
0 & 5 & 16 & 19 & 13 & 3 & 0 & 0 & 0 & 0 & 0 & 0 & 6.61 \\
0 & 0 & 0 & 15 & 11 & 10 & 8 & 6 & 2 & 0 & 0 & 0 & 4.04 \\
13 & 35 & 0 & 0 & 2 & 0 & 2 & 0 & 0 & 0 & 0 & 0 & 13.08 \\
0 & 0 & 0 & 5 & 3 & 13 & 5 & 3 & 0 & 0 & 0 & 0 & 2.14 \\
0 & 5 & 2 & 0 & 2 & 5 & 0 & 0 & 0 & 0 & 0 & 0 & 1.71 \\
13 & 10 & 15 & 10 & 0 & 0 & 0 & 2 & 0 & 0 & 0 & 0 & 10.57 \\
0 & 5 & 5 & 5 & 5 & 5 & 5 & 0 & 0 & 0 & 0 & 0 & 2.07 \\
0 & 2 & 5 & 8 & 6 & 6 & 8 & 0 & 0 & 0 & 0 & 0 & 3.34 \\
0 & 2 & 8 & 2 & 3 & 2 & 2 & 0 & 0 & 0 & 0 & 0 & 2.11 \\
66 & 8 & 8 & 10 & 3 & 2 & 0 & 0 & 0 & 0 & 0 & 0 & 31.95 \\
0 & 0 & 2 & 0 & 6 & 3 & 8 & 2 & 0 & 0 & 0 & 0 & 1.56 \\
0 & 2 & 2 & 2 & 3 & 11 & 0 & 0 & 0 & 0 & 0 & 0 & 1.79 \\
6 & 3 & 16 & 5 & 11 & 2 & 3 & 2 & 0 & 0 & 0 & 0 & 7.48
\end{tabular}

\section{5}

Rainfall

Crop Disease

Birds or Rats

Seed Avai lability

Fertilizer Availability

Crop Protection Chemicals

Credit

Irrigation Water Availability

Flooding

Labour Availabilty

Power for Land Preparation

Direct War Effects

Animal Diseases

Extension

Other Problems

$\begin{array}{rrrrrrrrrrrrr}1 & 2 & 3 & 4 & 5 & 6 & 7 & 8 & 9 & 10 & 11 & 12 & \text { Heighted Priority } \\ 19 & 15 & 6 & 15 & 0 & 0 & 0 & 0 & 5 & 0 & 0 & 0 & 14.15 \\ 0 & 6 & 26 & 16 & 6 & 2 & 2 & 0 & 2 & 0 & 0 & 0 & 7.66 \\ 0 & 0 & 3 & 15 & 11 & 3 & 0 & 2 & 0 & 0 & 0 & 0 & 3.31 \\ 0 & 0 & 0 & 0 & 6 & 11 & 11 & 0 & 0 & 3 & 0 & 0 & 2.19 \\ 5 & 44 & 3 & 6 & 5 & 0 & 0 & 0 & 0 & 0 & 0 & 0 & 13.01 \\ 0 & 0 & 0 & 2 & 3 & 6 & 0 & 2 & 0 & 0 & 0 & 0 & 1.00 \\ 0 & 0 & 6 & 0 & 2 & 2 & 2 & 2 & 0 & 0 & 2 & 0 & 1.43 \\ 6 & 23 & 21 & 3 & 0 & 2 & 0 & 0 & 0 & 0 & 0 & 0 & 11.09 \\ 0 & 0 & 2 & 0 & 0 & 5 & 0 & 0 & 2 & 2 & 0 & 0 & 0.72 \\ 0 & 0 & 2 & 3 & 3 & 8 & 5 & 5 & 5 & 0 & 0 & 0 & 2.22 \\ 6 & 2 & 3 & 5 & 5 & 2 & 0 & 0 & 2 & 0 & 0 & 0 & 4.71 \\ 60 & 8 & 2 & 13 & 5 & 5 & 0 & 3 & 2 & 0 & 0 & 0 & 30.00 \\ 0 & 2 & 3 & 6 & 2 & 2 & 0 & 0 & 2 & 0 & 0 & 0 & 1.83 \\ 0 & 0 & 0 & 0 & 8 & 0 & 11 & 8 & 2 & 3 & 0 & 0 & 2.03 \\ 2 & 2 & 16 & 2 & 5 & 3 & 5 & 3 & 0 & 0 & 0 & 0 & 4.64\end{array}$


TABLE 1-5(contd) - ANALYSIS OF GREATEST FARMING PROBLEMS - COMAUNITY SURVEY

PROVINCE OF KNR

1980 PERCENTAGE OF VILLAGES GIVING FOLLOWING PRIORITY TO PROBLEM

Rainfall

Crop Disease

Birds or Rats

Seed Availability

Fertilizer Availability

Crop Protection Chemicals

Credit

Irrigation Water Availability

Flooding

Labour Avail abilty

Power for Land Preparation

Direct Har Effects

Animal Diseases

Extension

Other Problems

\begin{tabular}{ccccccccccccc}
1 & 2 & 3 & 4 & 5 & 6 & 7 & 8 & 9 & 10 & 11 & 12 & Heighted Priority \\
\hline 0 & 3 & 0 & 0 & 0 & 0 & 0 & 0 & 0 & 0 & 0 & 0 & 1.61 \\
0 & 0 & 0 & 0 & 0 & 0 & 0 & 0 & 0 & 0 & 0 & 0 & 0.00
\end{tabular}

$\begin{array}{lllllllllllll}0 & 0 & 0 & 0 & 0 & 0 & 0 & 0 & 0 & 0 & 0 & 0 & 0.00 \\ 0 & 0 & 0 & 0 & 0 & 0 & 0 & 0 & 0 & 0 & 0 & 0 & 0.00\end{array}$

$\begin{array}{lllllllllllll}0 & 0 & 0 & 0 & 0 & 0 & 0 & 0 & 0 & 0 & 0 & 0 & 0.00 \\ 0 & 0 & 0 & 3 & 5 & 0 & 0 & 0 & 0 & 0 & 0 & 0 & 1.77\end{array}$

$\begin{array}{lllllllllllll}3 & 3 & 2 & 3 & 0 & 0 & 0 & 0 & 0 & 0 & 0 & 0 & 6.17\end{array}$

$\begin{array}{lllllllllllll}0 & 0 & 0 & 0 & 0 & 0 & 0 & 0 & 0 & 0 & 0 & 0 & 0.00\end{array}$

$\begin{array}{lllllllllllll}0 & 3 & 3 & 0 & 0 & 3 & 0 & 0 & 0 & 0 & 0 & 0 & 3.22\end{array}$

$\begin{array}{rrrrrrrrrrrrr}5 & 6 & 8 & 2 & 0 & 0 & 0 & 0 & 0 & 0 & 0 & 0 & 11.13\end{array}$

$\begin{array}{lllllllllllll}0 & 2 & 2 & 0 & 0 & 0 & 0 & 0 & 0 & 0 & 0 & 0 & 1.34\end{array}$

$\begin{array}{lllllllllllll}0 & 2 & 0 & 2 & 0 & 2 & 0 & 0 & 0 & 0 & 0 & 0 & 1.48\end{array}$

$\begin{array}{lllllllllllll}0 & 2 & 0 & 0 & 0 & 0 & 0 & 0 & 0 & 0 & 0 & 0 & 0.80\end{array}$

$\begin{array}{lllllllllllll}65 & 6 & 0 & 0 & 0 & 0 & 0 & 0 & 0 & 0 & 0 & 0 & 67.59\end{array}$

$\begin{array}{lllllllllllll}0 & 0 & 0 & 0 & 2 & 0 & 0 & 0 & 0 & 0 & 0 & 0 & 0.32\end{array}$

$\begin{array}{lllllllllllll}0 & 0 & 2 & 2 & 2 & 0 & 2 & 0 & 0 & 0 & 0 & 0 & 1.49\end{array}$

$\begin{array}{lllllllllllll}0 & 3 & 3 & 2 & 0 & 0 & 0 & 0 & 0 & 0 & 0 & 0 & \\ \end{array}$
Rainfall

Crop Disease

Birds or Rats

Seed Availability

Fertilizer Availability

Crop Protection Chemicals

Credit

Irrigation Water Availability

Flooding

Labour Availabilty

Power for Land Preparation

Direct War Effects

Animal Diseases

Extension

Other Problems

\begin{tabular}{ccccccccccccc}
1 & 2 & 3 & 4 & 5 & 6 & 7 & 8 & 9 & 10 & 11 & 12 & Heighted Priority \\
\hline 2 & 0 & 0 & 0 & 0 & 0 & 0 & 0 & 0 & 0 & 0 & 0 & 5.94 \\
0 & 0 & 0 & 0 & 0 & 0 & 0 & 0 & 0 & 0 & 0 & 0 & 0.00 \\
0 & 0 & 0 & 0 & 0 & 0 & 0 & 0 & 0 & 0 & 0 & 0 & 0.00 \\
0 & 0 & 5 & 2 & 0 & 0 & 0 & 0 & 0 & 0 & 0 & 0 & 7.43 \\
0 & 0 & 0 & 0 & 0 & 0 & 0 & 0 & 0 & 0 & 0 & 0 & 0.00 \\
0 & 0 & 0 & 0 & 0 & 0 & 0 & 0 & 0 & 0 & 0 & 0 & 0.00 \\
0 & 2 & 0 & 2 & 0 & 0 & 0 & 0 & 0 & 0 & 0 & 0 & 4.46 \\
15 & 3 & 0 & 0 & 0 & 0 & 0 & 0 & 0 & 0 & 0 & 0 & 59.41 \\
0 & 0 & 0 & 0 & 0 & 0 & 0 & 0 & 0 & 0 & 0 & 0 & 0.00 \\
0 & 2 & 0 & 0 & 0 & 0 & 0 & 0 & 0 & 0 & 0 & 0 & 2.97 \\
0 & 0 & 0 & 0 & 0 & 0 & 0 & 0 & 0 & 0 & 0 & 0 & 0.00 \\
2 & 3 & 2 & 0 & 0 & 0 & 0 & 0 & 0 & 0 & 0 & 0 & 13.86 \\
0 & 0 & 0 & 0 & 0 & 0 & 0 & 0 & 0 & 0 & 0 & 0 & 0.00 \\
0 & 0 & 0 & 0 & 0 & 0 & 0 & 0 & 0 & 0 & 0 & 0 & 0.00 \\
2 & 0 & 0 & 0 & 0 & 0 & 0 & 0 & 0 & 0 & 0 & 0 & 5.94
\end{tabular}

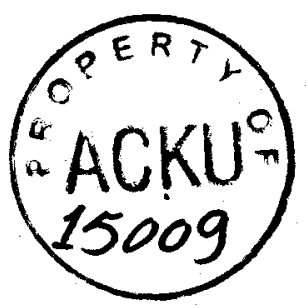

